DOE/NV/25946--1078

\title{
Glue film thickness measurements by spectral reflectance
}

\author{
B. R. Marshall, National Security Technologies, LLC, Special Technologies Laboratory, Santa \\ Barbara, California 93111, USA
}

\begin{abstract}
Spectral reflectance was used to determine the thickness of thin glue layers in a study of the effect of the glue on radiance and reflectance measurements of shocked-tin substrates attached to lithium fluoride windows. Measurements based on profilometry of the components were found to be inaccurate due to flatness variations and deformation of the tin substrate under pressure during the gluing process. The accuracy of the spectral reflectance measurements were estimated to be $\pm 0.5 \mu \mathrm{m}$, which was sufficient to demonstrate a convincing correlation between glue thickness and shock-generated light.
\end{abstract}

Measurement of film thickness using spectral reflectance is a well-known technique. A number of commercially available instruments use spectral reflectance for measurement of thickness and refractive index. ${ }^{1}$ These devices rely on the Fresnel reflections of the air/film and film/substrate interfaces. Measuring the thickness of a glue layer underneath a thick LiF window is difficult due to the very small $(\sim 0.1 \%)$ reflection from the window/glue interface. The reflection is small because the glue and $\mathrm{LiF}$ have similar indices of refraction. Also, nonuniform glue thickness requires a small measurement spot to achieve good fringe contrast. For the glue layer measurements described here, we constructed a measurement system consisting of a halogen lamp and a CCD spectrometer ${ }^{2}$ coupled via an uncollimated fiber optic probe to the surface under study (Figures 1 and 2) or an imaging probe (Figure 3). A typical sample (Figure 4) consists of a tin substrate, a few millimeters thick; a LiF window, several millimeters in thickness; and a thin layer of Loctite ${ }^{\circledR} 326$ glue $^{3}$ between them. On some samples, the tin surface was diamond-turned to a nearly specular finish, while other samples were considerably rougher.

The simple fiber optic probe consisted of two polished fibers mounted side by side in a stainless steel tube located approximately $25 \mathrm{~mm}$ from the window surface. The probe could theoretically receive light from any point on the surface within the fiber's numerical aperture. However, for relatively specular surfaces, only a fiber-sized spot on the surface actually contributes to the measurement. Although optically inefficient, this probe allows measurements through thick windows with minimal alignment difficulty. The high reflectivity of the tin substrate provided adequate signal levels at integration times of several hundred milliseconds.

A sample with a rough surface requires a small interrogation spot. Too large an area would include too much variation in thickness and the fringe contrast will be too low to make a measurement. For a rough surface, we used a multimode fiber optic $2 \times 2$ splitter to deliver light to a single 50-micron probe fiber and direct the return light to the spectrometer. A pair of lenses imaged the probe fiber onto the LiF surface at 5:1 magnification. The unused splitter connection was index matched and the probe fiber was angle polished to minimize backreflection. This 
single-fiber lens-relayed probe was used successfully on a very rough surface under a $19 \mathrm{~mm}$ thick LiF window. However, chromatic aberration in the lenses combined with dispersion in the window to produce a focus-dependent spectral artifact that has a signature similar to that of a submicron-thick glue layer. The probe optics will need to be chromatically corrected to measure very thin glue layers through very thick windows on rough surfaces.

The fiber mode structure affects the spatial distribution of light in the spectrometer, causing spectral artifacts that were comparable in amplitude to the interferometric modulation from the glue surfaces. To mitigate this problem, an active mode scrambler was used to vibrate the fiber and randomize the modes during data acquisition.

Light is reflected from both the $\mathrm{LiF} / \mathrm{glue}$ and glue/Sn surfaces. Ignoring multiple reflections, the intensity distribution of the sum of the two reflected waves is $I(\lambda)=I_{1}(\lambda)+I_{2}(\lambda)-2 \sqrt{I_{1}(\lambda) I_{2}(\lambda)} \cos (\delta(\lambda))$, where $I_{1}$ and $I_{2}$ are the intensities of the waves, $\lambda$ is the wavelength, and $\delta$ is the phase difference between them. ${ }^{4}$ Assuming a point source, a glue layer with planar surfaces, and a uniform refractive index, $n(\lambda)$, the optical path difference between the two reflections from a thin film is $\Delta P=2 n(\lambda) h \cos (\theta)$, where $\theta$ is the angle of incidence on the tin surface and $\mathrm{h}$ is the thickness of the glue layer. Therefore the phase difference for light reflected from the two surfaces is ${ }^{5} \delta=2 \pi \Delta P / \lambda=4 \pi n(\lambda) h \cos (\theta) / \lambda$. For a nondispersive medium (i.e., $n(\lambda)=n_{0}$ ) we observe that the frequency as a function of wavenumber, $k$, is a constant, $f(k)=4 \pi n_{0} h \cos (\theta)=f_{0}$. For our measurements the angle of incidence is nearly zero, and $\cos (\theta) \sim 1$, so the thickness $h=\frac{f_{0}}{4 \pi n_{0}}$. The frequency is determined by taking a Fourier transform of the interference spectrum followed by a Gaussian fit to find the center of the line. A more sophisticated analysis could potentially obtain the optical constants as well as the thickness of a thin film using a process of curve fitting and successive approximations. ${ }^{6}$

The refractive index of the glue was determined by measurement of a sample shown in Figure 5. A LiF window was attached to a mirror with a nominal $25 \mu \mathrm{m}$ thick annular spacer. A spot of glue occupied approximately the center $5 \mathrm{~mm}$ diameter of the aperture, and the glue was surrounded by an air gap. Measurements were made along the $\mathrm{x}$ and $\mathrm{y}$ axes, extending into the air gap region. This arrangement made it possible to detect any wedge or curvature in the spacing between the window and the mirror. The value for the refractive index of the glue obtained as the ratio of the optical path length in the glue to that in the air was $1.498 \pm .008$.

The success of the measurement depends on the index difference between the glue and the window material being large enough to generate a usable reflection. The refractive index of $\mathrm{LiF}$ is approximately 1.392 in the visible region, which gives a Fresnel reflection from the LiF/glue interface of 0.0014. Typical signals are shown in Figure 6. Window materials whose refractive index closely matches the glue will be much more difficult to measure.

We first collected a reference spectrum showing the system response of a bare, polished tin substrate. The glue and window have good transmission across the visible region of the spectrum used for the measurement, and therefore absorption can be neglected. The refractive index of the glue was assumed to be a constant over this spectral region. 
An example of a series of glue thickness measurements across a LiF/Sn target is shown in Figure 7. This sample consisted of a $1 \mathrm{~mm}$ thick $\mathrm{x} 40 \mathrm{~mm}$ diameter tin disk and a $10 \mathrm{~mm}$ thick x $38 \mathrm{~mm}$ diameter LiF window separated by a $40 \mu \mathrm{m}$ thick mechanical spacer. Across the diameter, the glue thickness varied to as low as $17 \mu \mathrm{m}$. Not shown on the graph is the glue layer thickness near the inner edge of the spacer, which was about $45 \mu \mathrm{m}$.

Table 1 summarizes the thickness measurements made in this series of experiments. For comparison, before assembly we measured the thicknesses of the tin and LiF at the center using either a digital or dial indicator. After they were glued, we measured the center thickness again with the same indicator and subtracted the thicknesses of the individual components. The results are listed in the "Glue Thickness Calculated" column. Note that the results are negative for some of the calculated glue thicknesses, which can easily occur when one of the materials is soft.

The measurement system was validated by measuring four reference standards obtained from Filmetrics Corp. These standards had thicknesses ranging from $2.91 \mu \mathrm{m}$ to $8.45 \mu \mathrm{m}$. The results were within $\pm 3 \%$ of the reference thickness supplied by the vendor. ${ }^{7}$

\section{Conclusion}

Spectral reflectance has been shown to work well for measuring glue thickness on shock targets with LiF windows. Improving the accuracy of thickness measurements will enhance our understanding of glue effects in shock measurements and will also provide valuable feedback for improving the window attachment process. The system could be optimized for future glue thickness measurements in several ways. The fiber-imaging probe could be optimized and corrected for dispersion. The data analysis could be expanded to include dispersion in the glue. Singlemode fiber could further reduce the spot size and eliminate speckle noise.

\section{Acknowledgements}

I would like to thank Mike Grover, Dale Turley, Gerald Stevens, David Holtkamp, and Lynn Veeser for suggesting this work and participating in the measurements.

This report was prepared as an account of work sponsored by an agency of the U.S. Government. Neither the U.S. Government nor any agency thereof, nor any of their employees, nor any of their contractors, subcontractors or their employees, makes any warranty or representation, express or implied, or assumes any legal liability or responsibility for the accuracy, completeness, or usefulness of any information, apparatus, product, or process disclosed, or represents that its use would not infringe privately own rights. Reference herein to any specific commercial product, process, or service by trade name, trademark, manufacturer, or otherwise, does not necessarily constitute or imply its endorsement, recommendation, or favoring by the U.S. Government or any agency thereof. The views and opinions of authors expressed herein do not necessarily state or reflect those of the U.S. Government or any agency thereof. 


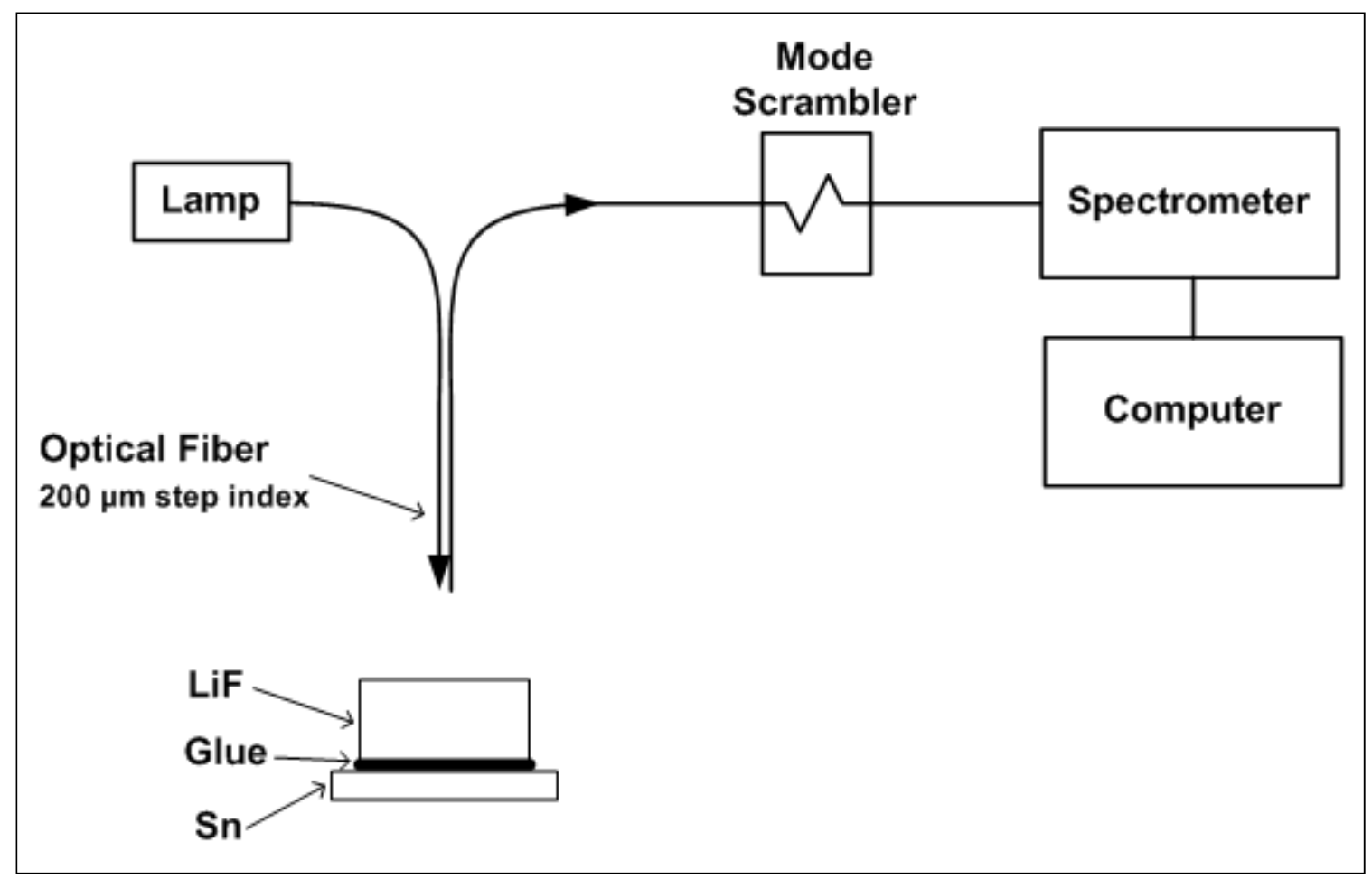

Figure 1. Schematic diagram of the spectral reflectance system with bare fiber probe

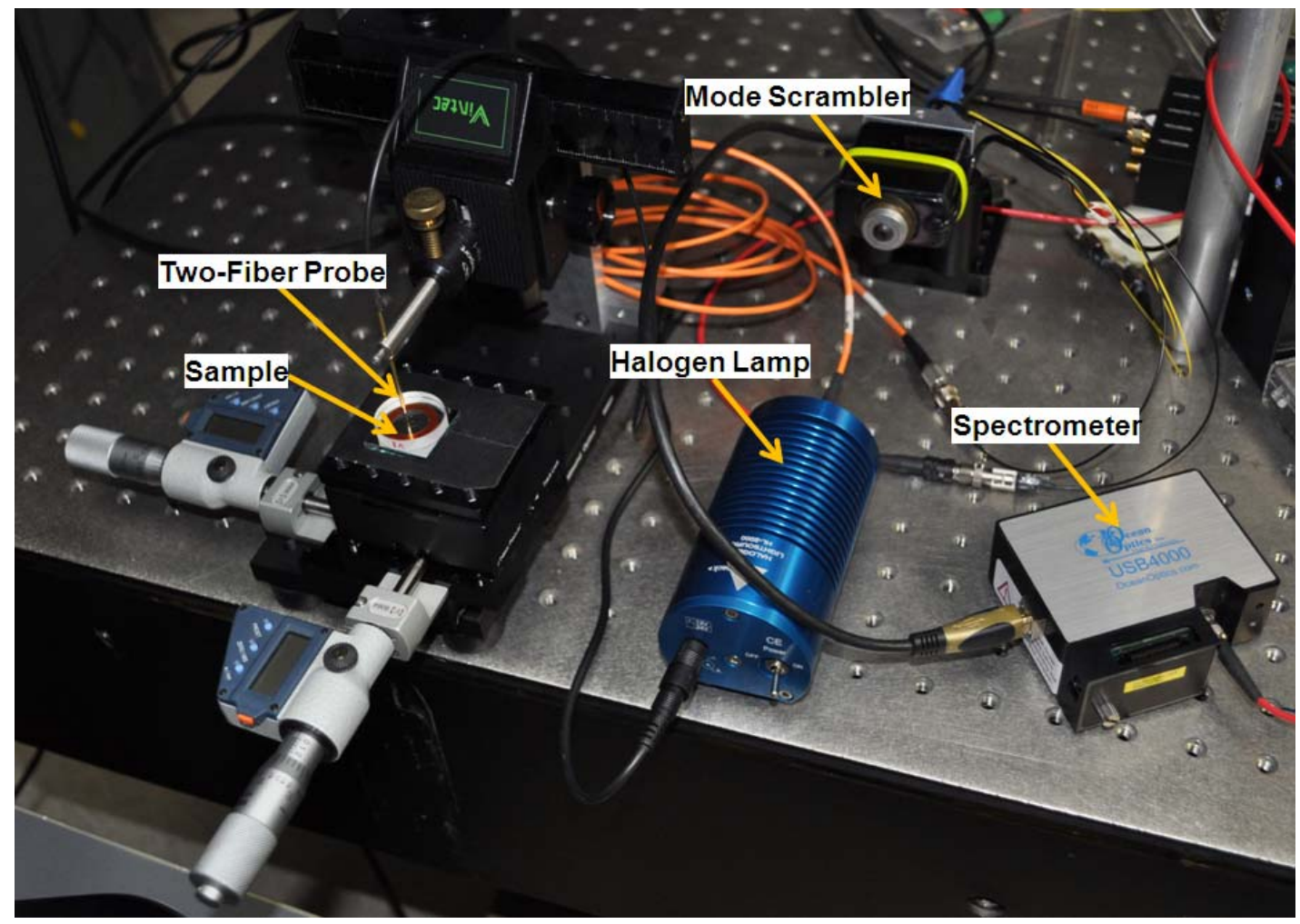

Figure 2. Glue thickness measurement system and probe 


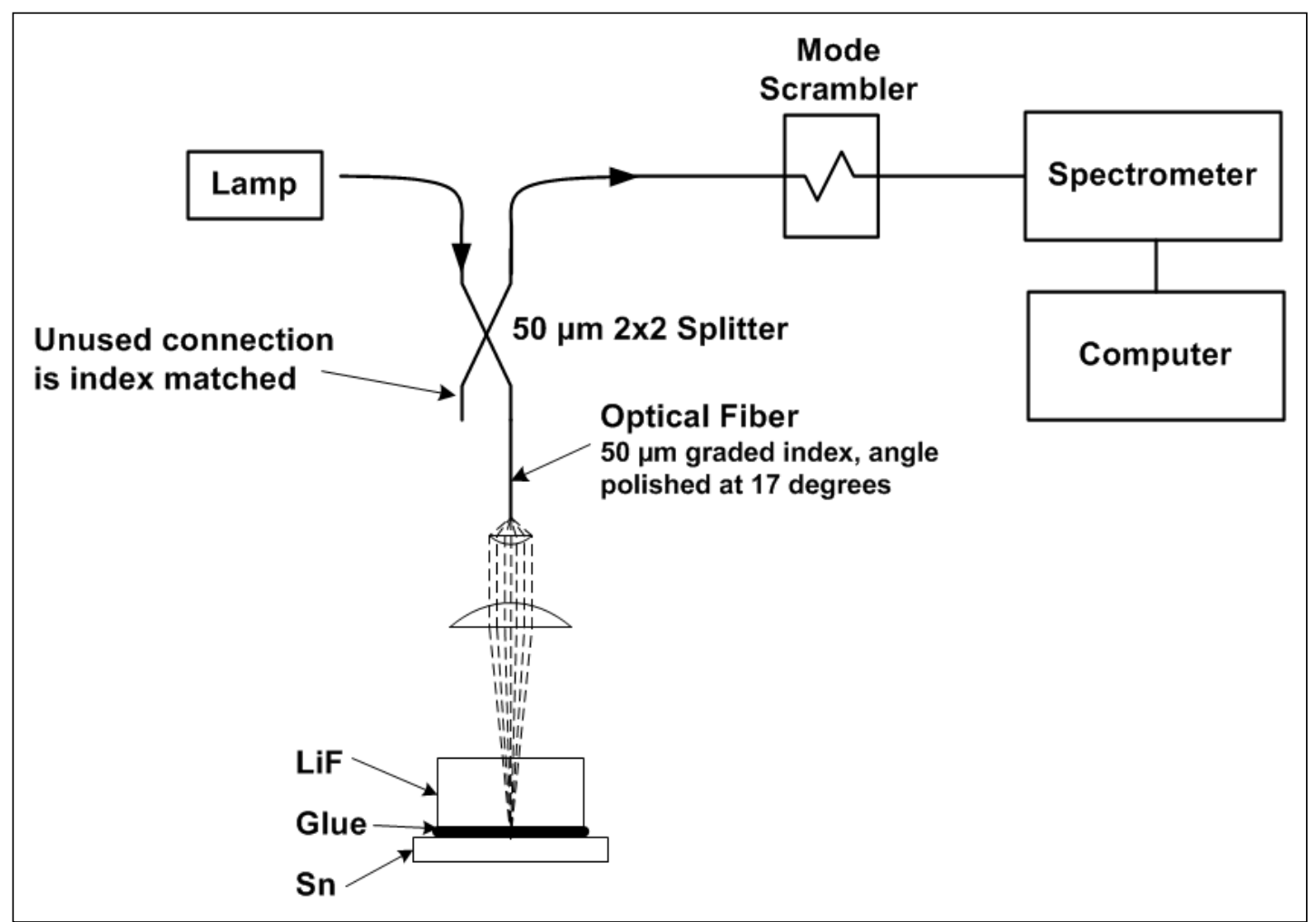

Figure 3. Schematic diagram of system with fiber-imaging probe

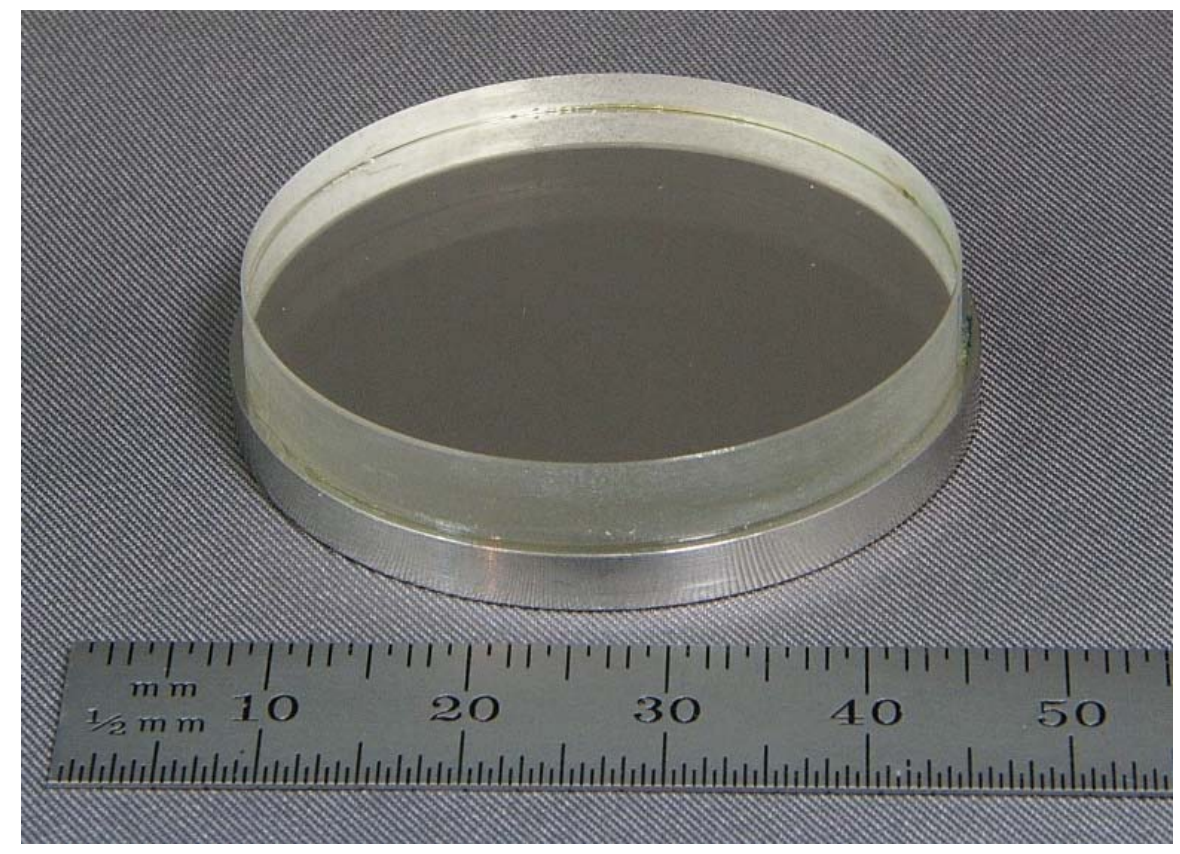

Figure 4. Typical sample with $2 \mathrm{~mm}$ thick tin and $3 \mathrm{~mm}$ thick lithium fluoride window 


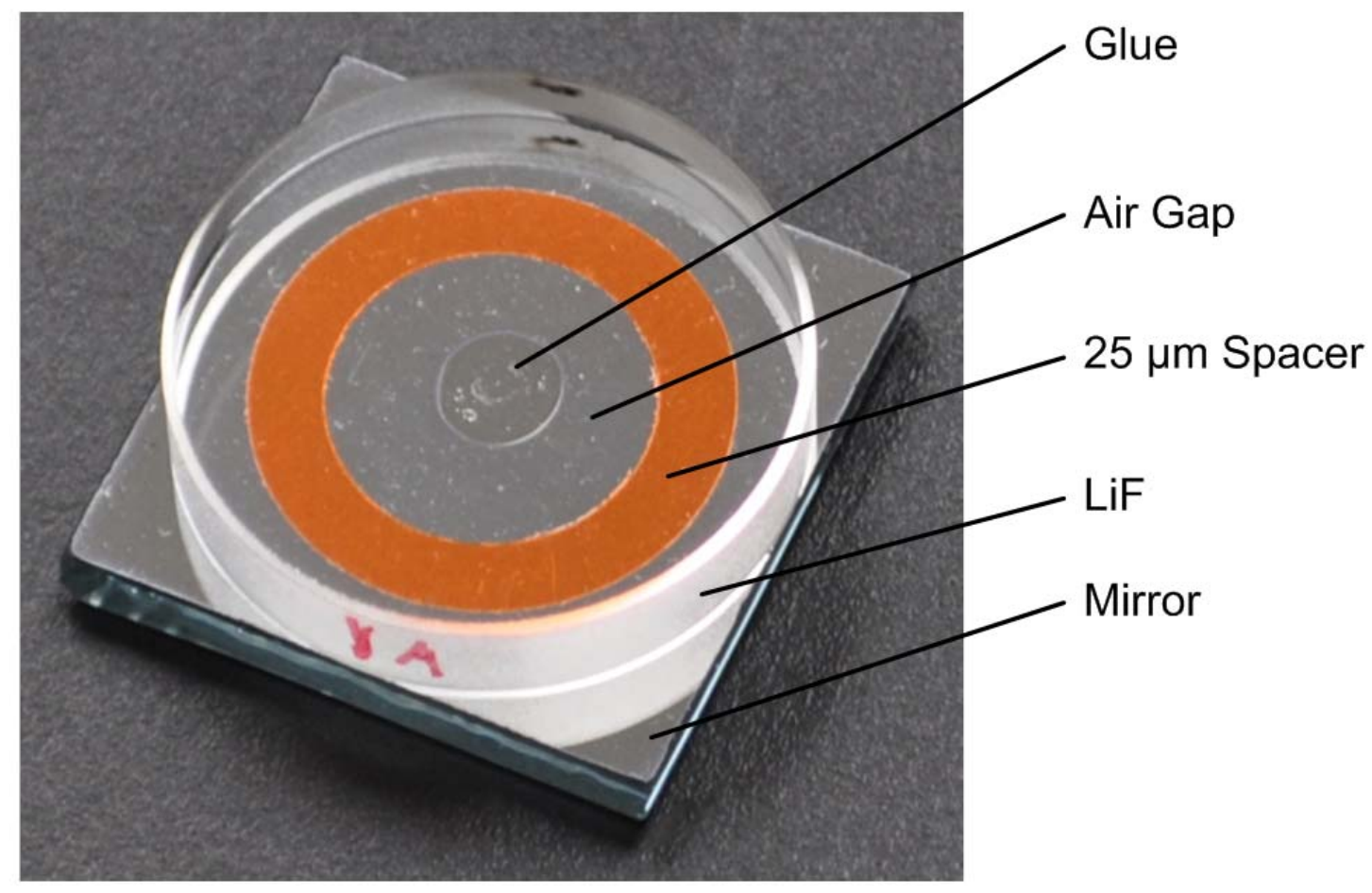

Figure 5. Sample for measuring the refractive index of the glue 

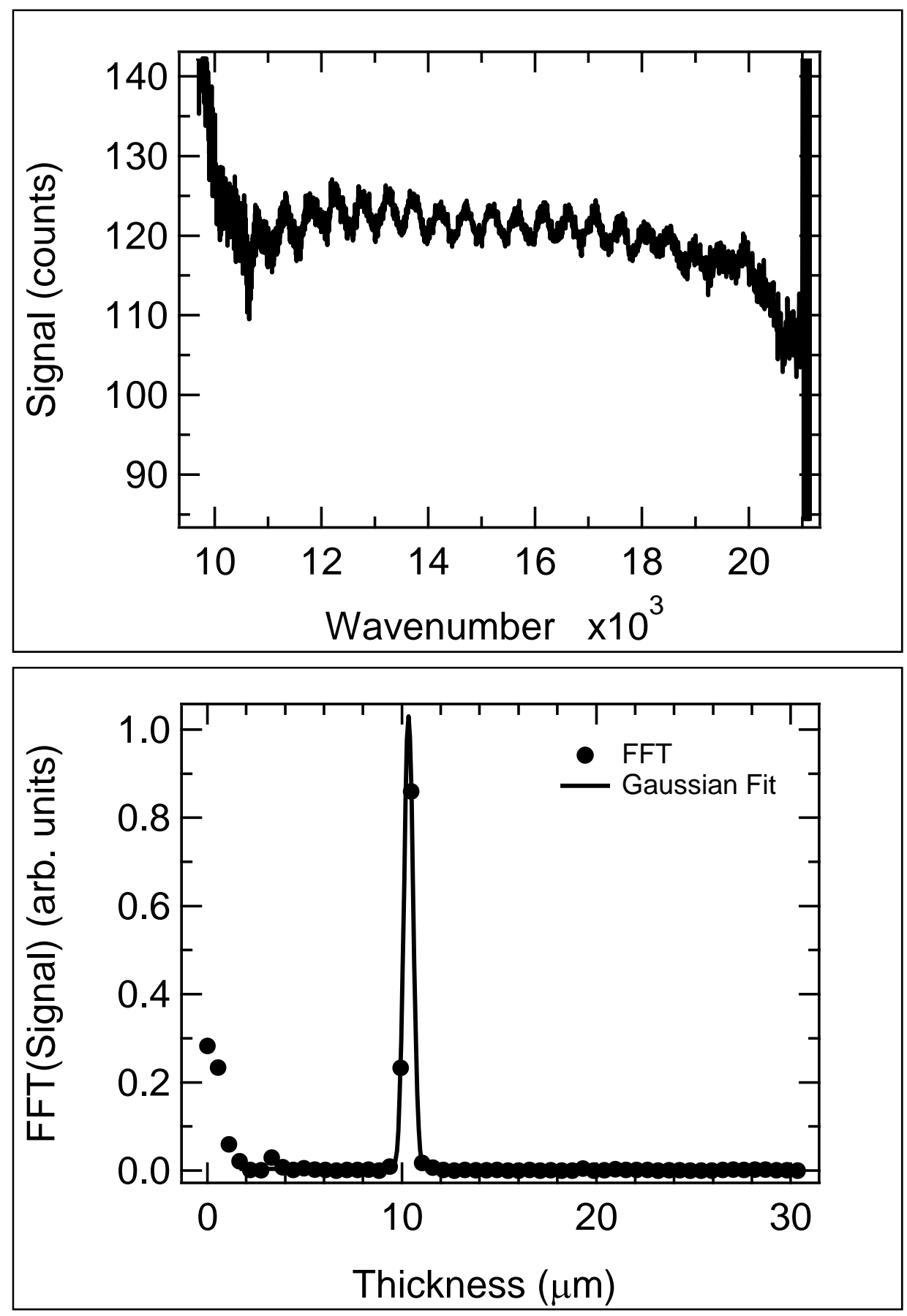

Figure 6. Typical signal (upper) and results of the Gaussian fit to the FFT that yields the thickness (lower). 

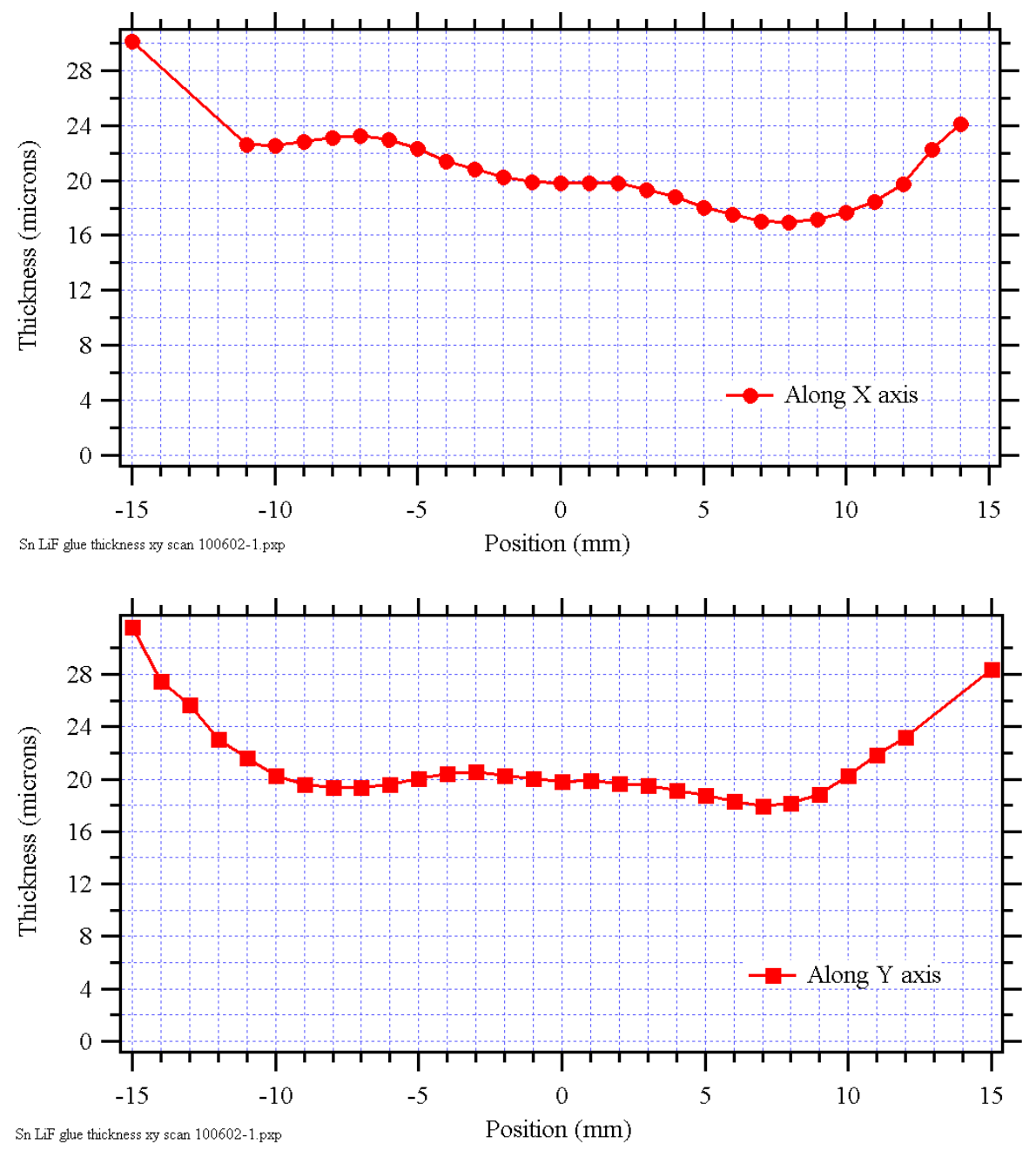

Figure 7. Measured glue layer thickness vs. position across the sample shown in Figure 4. 
Table 1. Measured results compared to results calculated from mechanical profilometry

\begin{tabular}{|c|c|c|c|c|c|c|}
\hline \multicolumn{7}{|c|}{2010 campaigns glued target list as of $5 / 10 / 10$} \\
\hline Campaign & Target \# & Sn cntr & LiF cntr & $\underset{\text { cntr }}{\text { Comb }}$ & $\begin{array}{c}\text { Glue } \\
\text { Thickness } \\
\text { Calculated } \\
(\mathbf{m m})\end{array}$ & $\begin{array}{c}\text { Glue } \\
\text { Thickness, } \\
\text { Interferometric } \\
\text { measurement } \\
\text { (mm) }\end{array}$ \\
\hline \multirow{5}{*}{ 造 } & 100504-1 & 0.976 & 5.948 & 6.925 & .001 & .0045 \\
\hline & $100504-2$ & 0.973 & 5.897 & 6.874 & .004 & .0033 \\
\hline & $100504-3$ & 0.970 & 5.936 & 6.931 & .025 & .0074 \\
\hline & $100504-4$ & 0.984 & 5.935 & 6.919 & No glue & .0037 \\
\hline & $100504-5$ & 0.987 & 5.750 & 6.737 & No glue & .0140 \\
\hline \multirow{3}{*}{$\frac{\bar{v}}{\frac{1}{s}}$} & $100505-1$ & 0.973 & 5.836 & 6.836 & .027 & .0057 \\
\hline & $100505-2$ & 0.906 & 5.778 & 6.631 & -.053 & .0011 \\
\hline & $100505-3$ & 0.931 & 5.762 & 6.609 & -.058 & .0028 \\
\hline \multirow{4}{*}{$\stackrel{\stackrel{\frac{0}{0}}{=}}{\frac{\frac{0}{0}}{n}}$} & 100506-1 & 3.494 & 5.086 & 8.574 & -.006 & .0073 \\
\hline & 100506-2 & 3.493 & 5.094 & 8.575 & -.012 & .0083 \\
\hline & $100506-3$ & 3.497 & 5.064 & 8.551 & -.010 & .0038 \\
\hline & 100506-4 & 3.495 & 5.070 & 8.561 & -.004 & .0090 \\
\hline \multirow{2}{*}{$\begin{array}{l}\text { LiF } \\
\text { sandwich }\end{array}$} & $100507-1$ & 1.629 & 5.990 & 7.610 & -.009 & .0069 \\
\hline & $100507-2$ & 1.823 & 5.977 & 7.801 & .001 & .0045 \\
\hline
\end{tabular}

\footnotetext{
${ }^{1}$ Filmetrics, Inc. San Diego, CA 92121, Angstrom Sun Technologies, Inc. Acton, MA 01720, Stellarnet, Inc. Tampa, Florida 33626

${ }^{2}$ Ocean Optics USB4000 spectrometer, HL2000 lamp

${ }^{3}$ Henkel Corporation, Düsseldorf, Germany

${ }^{4}$ (Born \& Wolfe, section 7.5.2 (eq.24))

${ }^{5}$ Young-Sik Ghim and Seung-Woo Kim, "Thin-film thickness profile and its refractive index measurements by dispersive white-light interferometry," Opt. Express 14, 11885-11891 (2006)

http://www.opticsinfobase.org/abstract.cfm?URI=oe-14-24-11885

${ }^{6}$ J. A. Dobrowolski, F. C. Ho, and A. Waldorf, "Determination of optical constants of thin film coating materials based on inverse synthesis," Appl. Opt. 22, 3191-3200 (1983)

http://www.opticsinfobase.org/abstract.cfm?URI=ao-22-20-3191

${ }_{7}^{7}$ Filmetrics, Inc. San Diego, CA 92121. Although Filmetrics does sell NIST traceable films, these films are not NIST traceable.
} 